\title{
ON SOME AMBIGUITIES OF THE ABSORPTION EDGE AND OPTICAL BAND GAPS OF AMORPHOUS AND POLYCRYSTALLINE SEMICONDUCTORS
}

This paper reports on somewhat puzzling character of the determination of the optical band gap energies of amorphous and polycrystalline semiconductors, where probably the mixed-phase composition and structural and compositional disorder are main reasons for the ambiguities. The analysis is performed using spectra of absorption coefficients of the featured samples of Si:H and Zn-Ti-O extracted by numerical analysis of transmittance measurements. The differences originating from using several procedures to process spectra of absorption coefficients are discussed.

Keywords: Absorption coefficient, thin film, microstructure, optical band gap, Tauc, Cody, Sokolov.

\section{Introduction}

For optoelectronic applications, fundamental optical absorption is a relevant issue. The absorption edge or the band edge is an optical property defined as the photon energy of the transition between the strong short- and the weak long-wavelength absorption in the spectrum of a semiconductor. The spectral position of this transition is determined by the energy separation between the valence and conduction bands of a material and corresponds to the threshold of a charge transition between the highest nearly filled band and the lowest nearly empty band. The optical bad gap is in general understood as the photon energy required for this transition.

Optical properties of amorphous and polycrystalline semiconductors have become objective of numerous studies, among all mainly for purposes of the photovoltaic conversion or other photonics applications. In general, amorphous semiconductors are materials with no long-range translational order of atomic sites [1 - 3]. However they often exhibit considerable short-range order of nanometer-sized crystallites. Polycrystalline (sometimes referred as microcrystalline) materials contain crystalline grains of random orientation separated by grain boundaries. Although the crystalline fraction commonly prevails, they may retain amorphous phase and inter- or intra-grain voids [4]. For this reason amorphous/polycrystalline semiconductors can possess the optical band gap which makes them similar to crystalline semiconductors. However an apparent widening of band tails of valence and conduction bands in amorphous and polycrystalline semiconductors leads to some controversies in characterizing the absorption edge and the optical band gap [5 - 7].

In this paper we describe several concepts of determining the optical band gap. Thin films of multi-phase hydrogenated silicon ( $\mathrm{Si}: \mathrm{H})$ and $\mathrm{Zn}-\mathrm{Ti}-\mathrm{O}$ ternary oxides are featured examples for comparing several attempts. From transmittance spectra the wavelength-dependent absorption coefficients are determined. The puzzle concerning optical band gaps estimated by various approaches is discussed.

\section{Band extrapolation methods}

It is well-known that a periodic potential typical for longrange ordered solids results in energy bands of sharp edges due to finite overlap integrals. Amorphous semiconductors are solids that do not possess the log-range order of atomic sites. Polycrystalline semiconductors consist of crystalline grains with different orientation and therefore the network is also ordered only in small regions. Additionally polycrystalline semiconductors may also contain amorphous phase and voids. Disorder typical for amorphous/polycrystalline semiconductors is followed by statistically varying widths and/or depths of potentials which result in broadening the edges into exponential tails. Other agents

\footnotetext{
* ${ }^{1}$ Jarmila Mullerova, ${ }^{2}$ Pavol Sutta

${ }^{1}$ Institute of Aurel Stodola, Faculty of Electrical Engineering, University of Zilina Liptovsky Mikulas, Slovakia

${ }^{2}$ New Technologies - Research Centre, University of West Bohemia, Plzen, Czech Republic

E-mail: mullerova@Im.uniza.sk
} 
to broaden the edges into tails may be material differences, microstructure development or quantum confinement effects.

Thus, new types of energy states, the so-called localized states appear in inorganic or organic amorphous and polycrystalline semiconductors (Fig. 1). The localized states are considered to be defect states due to disorder leading to the existence of over- and under-coordinated atoms and weak bonds. Besides the localized states in band tails, also deep states in the band gap exist due to dangling bonds, all of them responsible for many unique properties of amorphous/polycrystalline semiconductors [8]. The existence of band tail states has an important impact on their optical properties, especially on the band-to-band absorption crucial for many optoelectronic applications.

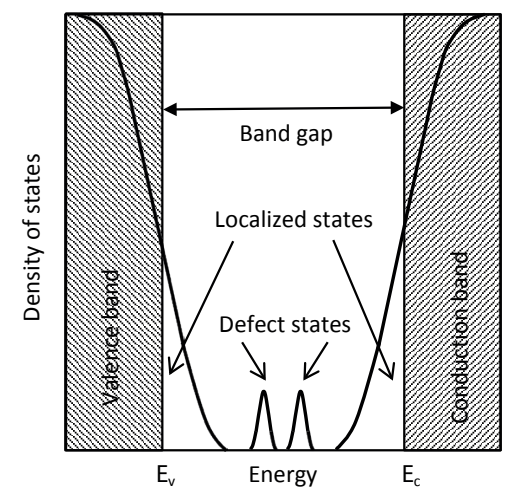

Fig. 1 Density of energy states in the vicinity of the edges of the conduction $\left(E_{c}\right)$ and valence band $\left(E_{v}\right)$ and in the band gap (localized states and deep states; the patterned rectangles represent the extended states

Then the optical band gap in amorphous/polycrystalline semiconductors characterizing the optical transitions between the extended states is a rather vague parameter owing to the localized states between valence and conduction bands. It is ambiguous to deduce the interval between extended states corresponding to the gap. A meaningful procedure to achieve knowledge on the band gap modifications is the inspection of the absorption spectrum of the material, i.e. the dependence of the absorption coefficient $\alpha$ on the wavelength $\lambda$ or on the photon energy $E$ related to $\lambda$ as $E=h c / \lambda,(h$ is the Planck's constant, $c$ is the speed of light $)$.

Typical optical absorption spectrum $\alpha(\lambda)$ of amorphous/ polycrystalline semiconductors with three apparent regions is in Fig. 2. The Urbach energy characterizes the tailing of the band edges that have an exponential energy distribution. It determines the slope of the exponential manner of the absorption coefficient described as $\alpha(E)=\alpha_{0} \exp \left[\left(E-E_{\mathrm{v}}\right) / E_{0}\right]$, where and $E_{\mathrm{v}}$ are constants and the Urbach energy $E_{0}$ is inversely proportional to the width of the exponential tail. It represents the tailing of the valence band density of states that is larger than the conduction band tailing. As the tail states are the direct figure of merit of the temperatureinduced, structural or compositional disorder, the Urbach energy is of considerable interest in a number of studies $[5,6]$. Due to the band tailing no sharp absorption threshold is obtained and the absorption edge cannot be clearly determined [2].

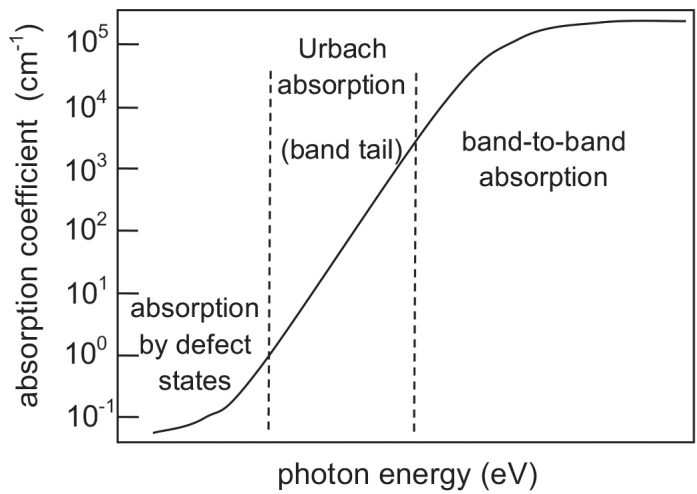

Fig. 2 The typical absorption coefficient of amorphous/polycrystalline semiconductors

Several representative methods have been reported to interpret the absorption region related to the optical transitions between extended states [7,9]. Assuming parabolic bands and constant matrix elements squared Jan Tauc derived the formula to describe the dependence of the absorption coefficient $\alpha$ on the photon energy $E[10]$

$(\alpha E)^{1 / 2}=B_{\text {Tauc }}\left(E-E_{g}^{\text {Tauc }}\right)$

where $E_{g}^{\text {Tauc }}$ is the so-called Tauc optical band gap energy. Hence the plot of $(\alpha E)^{1 / 2}$ versus the photon energy $E$ leads to a straight line whose intersection with the $E$-axis gives $E_{g}^{\text {Tauc }}$. The Tauc extrapolation has been widely adopted [11 - 13]. The scale factor $B_{\text {Tauc }}$ of the plot is often attributed to various aspects of semiconductor network. It includes information on the disorderinduced correlation of optical transitions between the valence and conduction bands and is often accepted to characterize the temperature and compositional disorder in amorphous and polycrystalline materials $[5,14]$.

Cody assumed the photon-energy dependent transition matrix element and the expression [15]

$(\alpha / E)^{1 / 2}=B_{\text {Cody }}\left(E-E_{g}^{\text {cody }}\right)$

The Cody plot $(\alpha / E)^{1 / 2}$ versus the photon energy usually obtains better linearity than the Tauc plot $(\alpha E)^{1 / 2}$. For this reason the Cody optical gap $E_{g}^{\text {Cody }}$ can be determined with less ambiguity than that from $(\alpha E)^{1 / 2}$ plot.

Sokolov studied optical gap fluctuations in amorphous Si and proposed the cubic root dependence

$(\alpha E)^{1 / 3}=B_{\text {Sokolov }}\left(E-E_{g}^{\text {Sokolov }}\right)$ 
The Sokolov model for the determination of the Sokolov band gap $E_{g}^{\text {Sokolov }}$ is similar to the model of the linear density of states suggested by Mott and Davis supposing a constant transition matrix element $[15,16]$. Similarly to the scale factor of the Tauc plot, $B_{\text {Cody }}$ and $B_{\text {Sokolov }}$ are the scale factors. Thus Cody, Tauc and Sokolov optical band gaps can be determined from extrapolation of the linear part of the plot to the energy axis. Linear parts of Cody plots are obviously more extended than Tauc plots. Hence the optical band gap determination could be more exact.

An alternative way to characterize the optical band gap is the iso-absorption gap $E_{03}\left(E_{04}\right)$ defining the photon energy at which the absorption coefficient achieves the value of $10^{3}\left(10^{4}\right)$ $\mathrm{cm}^{-1}$. Although none of them is reflecting particular differences in optical absorption, they are tolerable when the linear part of $(\alpha E)^{1 / 2}$ plot versus $E$ is reduced, especially in the presence of continuous distribution of localized states between the valence and conduction bands.

\section{Experimental determination of the absorption coefficient}

Absorption coefficients of transparent solids can be determined from transmittance/reflectance measurements. Transmittance $T(\lambda)$ of a homogeneous thin film of the thickness $d$ with parallel interfaces deposited on a thick substrate at nearly normal incidence of light is the following nonlinear function of $\lambda$, the refractive index $n_{1}$ and the extinction coefficient $k_{1}$ of the film and the refractive index $n_{2}$ of the non-absorbing substrate [17]

$$
T(\lambda)=\frac{A x}{B-C x+D x^{2}}
$$

where the absorbance $x=\exp (-\alpha d)$. Equation (4) is obtained by summing the multiply-transmitted waves. Here

$$
\begin{aligned}
A= & 16 n_{2}\left(n_{1}^{2}+k_{1}^{2}\right) \\
B= & {\left[\left(1+n_{1}\right)^{2}+k_{1}^{2}\right]\left[\left(n_{1}+n_{2}^{2}\right)\left(n_{1}+1\right)+k_{1}^{2}\right] } \\
C= & 2 \cos \varphi\left[\left(n_{1}^{2}+k_{1}^{2}-1\right)\left(n_{1}^{2}+k_{1}^{2}-n_{2}^{2}\right)-\right. \\
& \left.-2 k_{1}^{2}\left(n_{1}^{2}+1\right)\right]-2 \sin \varphi\left[\left(n_{1}^{2}+k_{1}^{2}-1\right)\right. \\
& \left.\left(n_{2}^{2}+1\right)+2\left(n_{1}^{2}+k_{1}^{2}-n_{2}^{2}\right)\right] \\
D= & {\left[\left(n_{1}-1\right)^{2}+k_{1}^{2}\right]\left[\left(n_{1}-1\right)\left(n_{1}-n_{2}^{2}\right)+k_{1}^{2}\right] }
\end{aligned}
$$

The phase factor is defined as $\varphi=4 \pi n_{1} d / \lambda$. The absorption coefficient $\alpha$ (Fig. 2) of the film corresponding to band-to-band transitions is related to $k_{1}$ and $\lambda$ through the relation

$$
\alpha=4 \pi k_{1} / \lambda
$$

Theoretical transmittance $T(\lambda)$ to be compared with the experiment can be calculated by Equation (4) using the proper dispersion relations of $n_{1}(\lambda), k_{1}(\lambda)$. Currently employed $n_{1}(\lambda), k_{1}(\lambda)$ of amorphous/polycrystalline semiconductors are featured by the
Tauc-Lorentz or the Cody-Lorentz models [18 - 21]. Refractive indices $n_{1}$ and extinction coefficients $k_{1}$ of the films as real and imaginary parts of the complex refractive index $\tilde{n}_{1}=n_{1}+i k_{1}$ can be extracted from the numerical comparison $T(\lambda)$ and measured transmittance $T_{\text {exp }}(\lambda)$. The fitting procedure minimizes deviations between experimental $T_{\text {exp }}(\lambda)$ and theoretical $T(\lambda)$ in the broad spectral region including the vicinity of the absorption edge. To calculate $n_{1}(\lambda), k_{1}(\lambda)$ by applying an optimization procedure based on genetic algorithm is a proper tool [22]. The absorption coefficient will be known from Equation (5).

Reflectance of the film can be expressed similarly by summing the multiply-reflected waved and can be used for the determination of $n_{1}(\lambda), k_{1}(\lambda)$ when the film is deposited on an absorbing substrate [23].

\section{Featured examples}

Two unalike semiconductors of different compositional order and structure and both of extensive applications in optoelectronics and photovoltaics were used to illustrate the means of the determination of optical band gaps. The series of Si:H and ternary oxides of Zn-Ti-O were deposited on clean Corning Eagle 2000 glass substrates (details here [24, 25]). Si:H is recognized for extensive applications in solar cells, thin film transistors, LEDs, display and imaging technologies etc. In addition to these applications $\mathrm{Zn}-\mathrm{Ti}-\mathrm{O}$ is also known as a semiconducting photocatalyst.

1. A series of Si:H (the thickness of approx. $400 \mathrm{~nm}$ ) was deposited by parallel plate PECVD. The depositions differ by the amount of additional hydrogen $\left(\mathrm{H}_{2}\right)$ added to silane $\left(\mathrm{SiH}_{4}\right)$ plasma. The $\mathrm{H}_{2}$ to $\mathrm{SiH}_{4}$ flows ratio is the so-called dilution $R$ ranging from $R=10$ to $R=70$. In our previous paper [24] multi-phase composition was detected. The samples deposited at $R<30$ were identified as amorphous $\mathrm{Si}$ :H with a certain volume fraction of voids, samples at $R \geq 30$ converted to polycrystalline with certain fractions of voids and amorphous phase.

2. A series of zinc titanium oxide $\mathrm{Zn-Ti-O}$ was prepared by reactive magnetron co-sputtering in reactive mode. Seven samples of the thickness of $200-300 \mathrm{~nm}$ were deposited with increasing atomic percentage Ti content [25]. With increasing Ti content not only compositional changes but also the evolution of a complicated polycrystalline structure of the decreasing crystallite size and the preferred orientation of crystallites in a certain direction against the surface occurred. The sample of the highest Ti content (12.5\%) was identified as amorphous.

Structural properties (Table 1) were analysed by X-ray diffraction using an automatic powder diffractometer $\mathrm{X}^{\prime}$ Pert Pro with $\mathrm{CuK} \alpha$ radiation supplemented by several microstructuredevoted methods (Raman and FTIR spectroscopy, HR-TEM, 


\section{COMMNICOIIONS}

Microstructure details of the featured samples and $B_{\text {Tauc }}$ factors. $\mathrm{p}_{\mathrm{c}} / \mathrm{p}_{\mathrm{a}}[\%]$ - the ratio of the crystalline to amorphous volume fraction in Si:H determined by XRD, pc- polycrystalline structure, prevailing phase of Zn-Ti-O indicated

\begin{tabular}{ccc}
\hline & Si:H & \\
\hline$R$ & $p_{c} / p_{a}[\%]$ & $\begin{array}{c}B_{\text {Tauc }} \\
{\left[\mathrm{eV}^{-1 / 2} \mathrm{~cm}^{-1 / 2}\right]}\end{array}$ \\
10 & 0 & 670 \\
20 & 0 & 580 \\
30 & 0 & 630 \\
40 & 54 & 370 \\
50 & 63 & 370 \\
60 & 75 & 320 \\
70 & 77 & 330 \\
\hline
\end{tabular}

\begin{tabular}{|c|c|c|}
\hline \multicolumn{3}{|c|}{ Zn-Ti-O } \\
\hline $\begin{array}{c}\mathrm{Ti} \\
\text { (at. \%) }\end{array}$ & $\begin{array}{l}\text { Structure, composition and prevailing orientation of } \\
\text { crystallites }\end{array}$ & $\begin{array}{l}B_{\text {Tauc }} \\
{\left[\mathrm{eV}^{-1 / 2} \mathrm{~cm}^{-1 / 2}\right]}\end{array}$ \\
\hline 0 & $p c,(002) \mathrm{ZnO}$ & 1860 \\
\hline 1.1 & $p c,(002) \mathrm{ZnO}$ & 1500 \\
\hline 2.4 & $p c,(002),(101) \mathrm{ZnO}$, bimodal distribution of crystallites & 1200 \\
\hline 5.2 & $\begin{array}{l}p c,(104) \mathrm{ZnTiO}_{3},(004) \mathrm{TiO}_{2} \text { atanas, bimodal } \\
\text { distribution of crystallites }\end{array}$ & 1140 \\
\hline 6.7 & $\begin{array}{l}p c,(104) \mathrm{ZnTiO}_{3},(004) \mathrm{TiO}_{2} \text { atanas, bimodal } \\
\text { distribution of crystallites }\end{array}$ & 1050 \\
\hline 8.7 & $\begin{array}{l}p c,(104) \mathrm{ZnTiO}_{3},(004) \mathrm{TiO}_{2} \text { atanas, bimodal } \\
\text { distribution of crystallites }\end{array}$ & 950 \\
\hline 12.5 & amorphous & 1000 \\
\hline
\end{tabular}

EEDS). Optical transmittances recorded by Specord 210 spectrophotometer [24,25] were used to calculate $\alpha$ (Figs. 3 and 4)

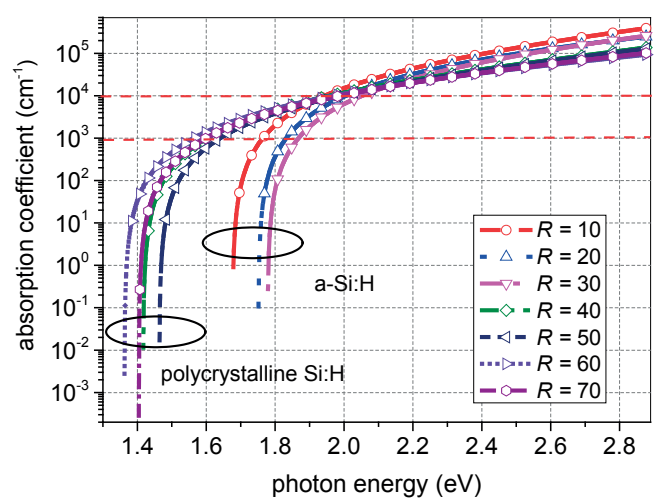

Fig. 3 Absorption coefficients of Si:H (red dash lines: iso-absorption levels of $10^{3} \mathrm{~cm}^{-1}, 10^{4} \mathrm{~cm}^{-1}$ )

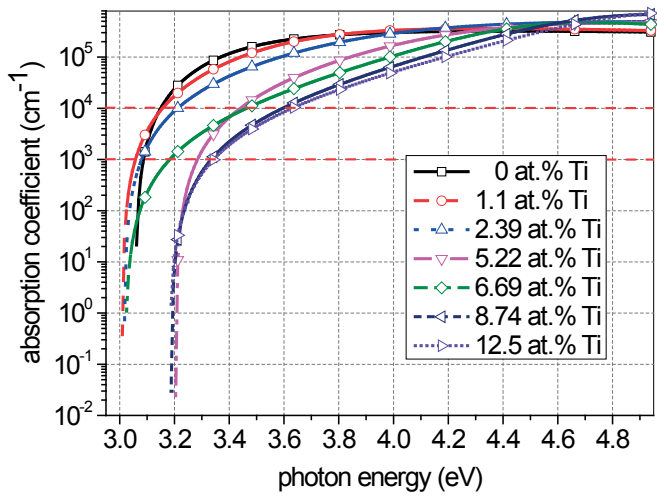

Fig. 4 Absorption coefficients of $\mathrm{Zn}-\mathrm{Ti}-\mathrm{O}$ (red dash lines: iso-absorption level of $\left.10^{3} \mathrm{~cm}^{-1}, 10^{4} \mathrm{~cm}^{-1}\right)$
From Fig. 3 the iso-absorption gaps $E_{03}, E_{04}$ of Si:H were directly deduced. The iso-absorption gap $E_{03}$ cannot be considered from Fig. 4 for Zn-Ti-O because it is too close to the Urbach absorption. $E_{g}^{\text {Tauc }}, E_{g}^{\text {Cody }}$ and $E_{g}^{\text {Sokolov }}$ gaps were determined using plots corresponding to Equations (1), (2) and (3). Representative Tauc and Cody plots of Si:H and Zn-Ti-O are in Figs. 5 - 8. Some of linear extrapolations are indicated by dash lines. Sokolov plots return the least linear parts for the optical band gap determination.

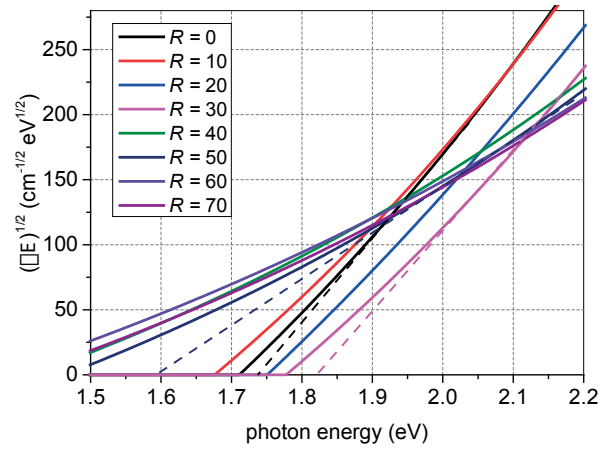

Fig. 5 Tauc plots of Si:H

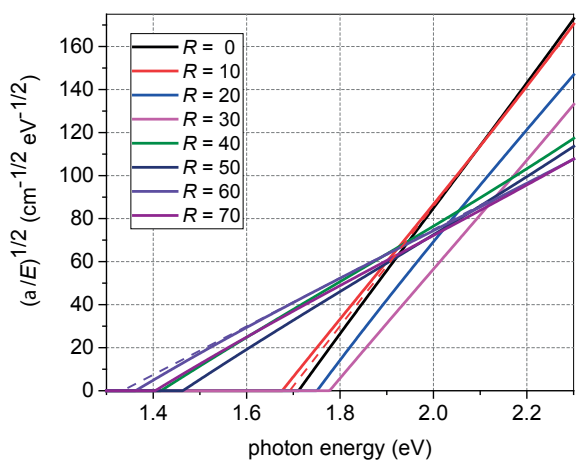

Fig. 6 Cody plots of Si:H 


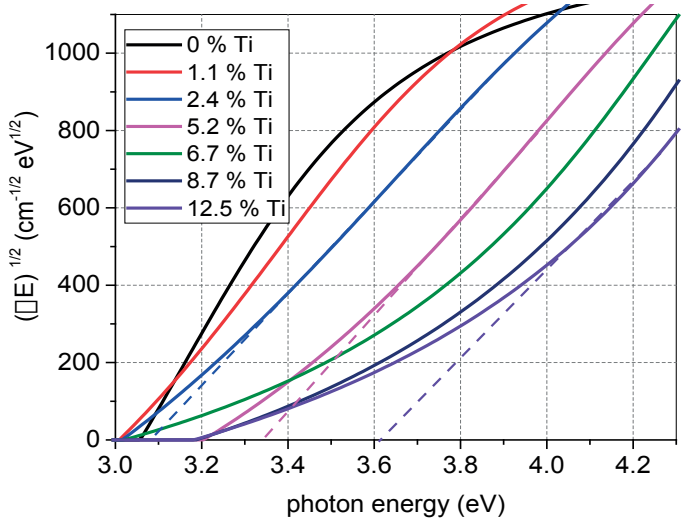

Fig. 7 Tauc plots of $\mathrm{Zn-Ti-O}$

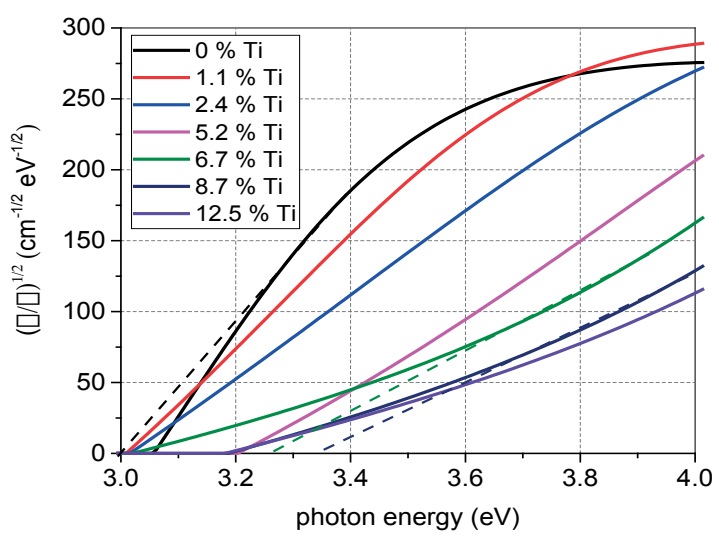

Fig. 8 Cody plots of Zn-Ti-O

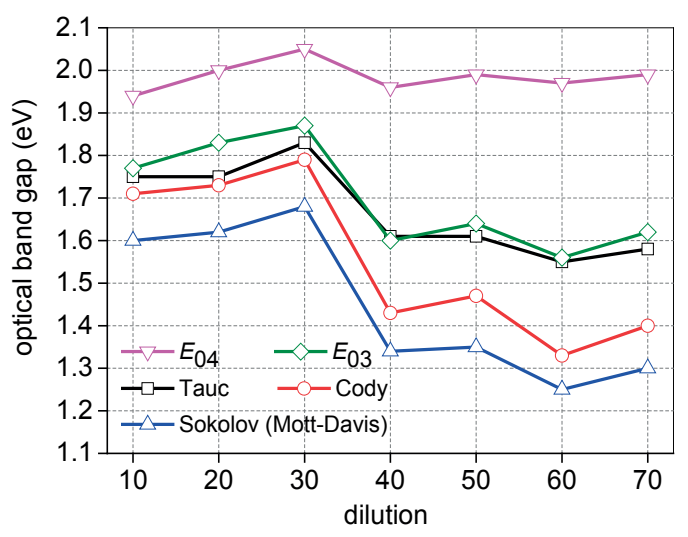

Fig. 9 Optical band gaps of Si:H deposited at different hydrogen dilution

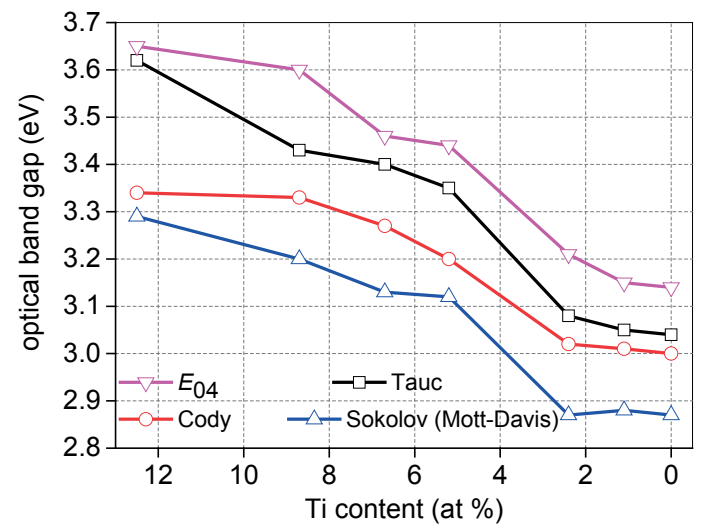

Fig. 10 Optical band gaps of $\mathrm{Zn}$-Ti-O versus decreasing Ti content

The following conclusions can be made from using several approaches. Zn-Ti-O is apparently a wider-gap semiconductor than Si:H. It is obvious that Cody plots of $\mathrm{Si}: \mathrm{H}$ have more extended linear parts than Tauc. Therefore the Cody band gaps can be determined in less ambiguity than Tauc. In case of $\mathrm{Zn}$-Ti-O neither Tauc nor Cody extrapolations seem to be more linear. We see that for both materials $E_{g}^{\text {Sokolov }}<E_{g}^{\text {Cody }}<E_{g}^{\text {Tauc }}$ is valid (Figs. 9 and 10). $E_{g}^{\text {Sokolov }}, E_{g}^{\text {Cody }}, E_{g}^{\text {Tauc }}$ are decreasing functions of growing dilution in Si:H and dropping Ti content in $\mathrm{Zn}$-Ti-O. It is not difficult to disclose that $E_{04}$ is always larger than $E_{g}^{\text {Tauc }} . E_{03}$ coexists well with $E_{g}^{\text {Tauc }}$ for $\mathrm{Si}: \mathrm{H}$.

$B_{\text {Tauc }}$ compares well with the structural disorder in $\mathrm{Si}: \mathrm{H}$ and compositional disorder in $\mathrm{Zn}$-Ti-O (Table 1). Larger values of $B_{\text {Tauc }}$ were found in amorphous $\mathrm{Si}: \mathrm{H}$ in contrast to the polycrystalline $\mathrm{Si}: \mathrm{H}$ with randomly oriented polycrystalline grains separated by grain boundaries. Surprisingly this is not the case of Zn-Ti-O in which both the increasing compositional disorder and more complex polycrystalline structure with growing Ti content may be the origin of decreasing $B_{\text {Tauc }}$. Moreover Zn-Ti-O samples with increasing $\mathrm{Ti}$ content manifest decreasing crystallite size that could indicate the quantum size effect and be followed by the decrease of $B_{\text {Tauc }}$.

\section{Conclusions}

Knowledge of optical absorption is a relevant issue for all light-based applications of amorphous/polycrystalline semiconductors. However due to the considerable widening of band tails of the valence and conduction bands the optical band gap is not an unambiguous property. Several approaches of the band gap determination are described. As we demonstrate in case of thin films of Si:H and $\mathrm{Zn}-\mathrm{Ti}-\mathrm{O}$ with different structural and compositional disorder no general rule exists to select the proper band extrapolation. For a specific material we recommend using the model resulting in a linear trend in a larger energy range 
and thus in less uncertainty in determining optical band gaps. Accordingly as Tauc, Cody and Sokolov optical band gaps are considerably dissimilar it should be always obligatory to comment on the applied extrapolation.

\section{Acknowledgements}

This work was partly supported by the Slovak Grant Agency under the project VEGA 2/0076/15 and by the Slovak Research and Development Agency under the projects APVV-15-0152, APVV-0888-12. The results were partly developed within the CENTEM project, reg. no. CZ.1.05/2.1.00/03.0088 co-funded by the ERDF within the OP RDI programme, and in the follow-up sustainability stage, supported through CENTEM+ (LO1402) by financial means from the Ministry of Education, Youth and Sports of the Czech Republic under the National Sustainability Programme I.

\section{References}

[1] SHIMAKAWA, K., SINGH, J., O’LEARY, S. K.: Optical Properties of Condensed Matter and Applications. Singh, J. (Ed.), John Wiley and Sons, Chichester 2007.

[2] CHOPRA, K. L., PAULSON, P. D., DUTTA, V.: Thin Film Solar Cells: An Overview. Progress in Photovoltaics: Research and Applications, 12, 69-92, 2004.

[3] SHAH, A.: Thin-Film Silicon Solar Cells. EPFL Press, Lausanne, 2010.

[4] MULlerovA, J., SUTTA, P., VAN ELZAKKER, G., ZEMAN, M., MIKULA, M.: Microstructure of Hydrogenated Silicon Thin Films Prepared from Silane Diluted with Hydrogen. Applied Surface Science, 254, 3690-3695, 2008.

[5] LIU, P., LONGO, P., ZASLAVSKY, A., PACIFICI, D.: Optical Band Gap of Single-Nd Multi-Layered Amorphous Germanium Ultra-Thin Films. Journal of Applied Physics, 119, 014304, 2016.

[6] ORAPUNT, F., O'LEARY, S.: A Quantitative Characterization of the Optical Absorption Spectrum Associated with Hydrogenated Amorphous Silicon. Journal of Materials Science: Materials in Electronics, 20, 1033 - 1038, 2009.

[7] BERSCH, M. D., DIEBOLD, A. C., COSIGLIO, S., CLARK, R. D., LEUSINK, G. J., KAACK, T.: Comparison of Methods to Determine Bandgaps of Ultrathin $\mathrm{HfO}_{2}$ Films Using Spectroscopic Ellipsometry. Journal of Vacuum Science \& Technology A, A29(4), 041001, 2011.

[8] JURECKA, S., JAMNICKY, I.: Study of the Density Of States Distribution in the $\mathrm{SiO}_{2} / \mathrm{Si}$ Structure. Communications Scienfitic Letters of the Universtiy of Zilina, 12(2), 58-61, 2010.

[9] MOK, T. M., O’LEARY, S. K.: The Dependence of the Tauc and Cody Optical Gaps Associated with Hydrogenated Amorphous Silicon on the Film Thickness: $\alpha$ l Experimental Limitations and the Impact of Curvature in the Tauc and Cody Plots. Journal of Applied Physics, 102, 113525, 2007.

[10] TAUC, J. (Ed.): Amorphous and Liquid Semiconductors. Plenum, New York, 1976.

[11] VIEZBICKE, B. D., PATEL, S., DAVIS, B. E., BIRNIE III, D. P.: Evaluation of the Tauc Method for Optical Absorption Edge Determination: ZnO thin Films as a Model System. Physica Status Solidi B, B252(8), 1700-1710, 2015.

[12] KODOLBAS, A. O.: Empirical Calibration of the Optical Gap in a-Si ${ }_{1-x} C_{x}: \mathrm{H}(\mathrm{x}<0.20)$ Alloys. Materials Science and Engineering: B, B98, 161-166, 2003.

[13] GUERRA, J. A., MONTANEZ, L. M., TUCTO, K., ANGUlO, J.: Bandgap Engineering of Amorphous Hydrogenated Silicon Carbide. MRS Advances, 1(43), 2929-2934, 2016.

[14] COSENTINO, S., MIRITELLO, M., CRUPI, I., NICOTRA, G., SIMONE, F., SPINELLA, C., TERRASI, A., MIRABELLA, S.: Room-Temperature Efficient Light Detection by Amorphous Ge Quantum Wells. Nanoscale Research Letters, 8, 128, 2013.

[15] SINGH, J., SHIMAKAWA, K., KOTLIAR, G., TOKURA, Y.: Advances in Condensed Matter Science. Taylor\&Francis, London, New York, 2003.

[16] SRIVASTAVA, S., MEHTA, N., TIWARI, R. S., SHUKLA, R. K., KUMAR, A. J.: Chemical Bond Approach to Optical Band Gap in Se100-xSb x Chalcogenide Glasses. Journal of Optoelectronics \& Advanced Materials, 13(1), 13-18, 2011.

[17] SWANEPOEL, R.: Determination of the Thickness and Optical Constants of Amorphous Silicon. Journal of Physics E: Scientifics Instruments, 16, 1214-1222, 1983.

[18] JELLISON Jr., G. E., MODINE, F. A.: Parametrization of the Optical Functions of Amorphous Materials in the Interband Region. Applied Physics Letters, 69(3), 371-373, 1996 and erratum Applied Physics Letters, 69(14), 2137, 1996. 
[19] PRICE, J., HUNG, P. Y., RHOAD, T., FORAN, B., DIEBOLD, A. C.: Spectroscopic Ellipsometry Characterization of HfxSiyOz Films Using the Cody-Lorentz Parameterized Model. Applied Physics Letters, 85(10), 1701 - 1703, 2004.

[20] FERlaUtO, A. S., FerreirA, G. M., PEARCE, J. M., WrOnSKI, C. R., COllinS, R. W., DENG, X., GANGULY, G.: Analytical Model for the Optical Functions of Amorphous Semiconductors from the Near-Infrared to Ultraviolet: Applications in Thin Film Photovoltaics. Journal of Applied Physics, 92(5), 2424-2436, 2002.

[21] LIKHACHEV, D. V., MALKOVA, N., POSLAVSKY, L.: Modified Tauc-Lorentz Dispersion Model Leading to a More Accurate Representation of Absorption Features Below the Bandgap. Thin Solid Films, 589, 844-851, 2015.

[22] MUllerova, J., PRUSAKOVA, L., NETRVAlOVA, M., VAVRUNKOVA, V., SUTTA, P.: A Study of Optical Absorption in Amorphous Hydrogenated Silicon Thin Films of Varied Thickness. Applied Surface Science, 256, 5667 - 5671, 2010.

[23] JAFAR ABDUL-GADER, M.M.: Comprehensive formulations for the total normal-incidence optical reflectance and transmittance of thin films laid on thick substrates. European International Journal of Science and Technology, 2, 214 - 274, 2013.

[24] MUlleroVA, J., SUTTA, P., PRUSAKOVA, L., NETRVALOVA, M.: Dispersive and BEMA Investigation on Optical Properties of Photovoltaic Thin Filas. Proceeding of SPIE, 9441, 94411J, 2014.

[25] MUlLerova, J., SUTTA, P., PRUSAKOVA, L., NETRVAlOVA, M.: Optical Properties of Zinc Titanate Perovskite Prepared by Reactive rf Sputtering. Submitted to Journal Electrical Engineering, 2017. 